\title{
NHS Clinical Knowledge Summaries
}

\author{
Derek Richards \\ Centre for Evidence-based Dentistry, Oxford, UK
}

The UK National Health Service (NHS) Clinical Knowledge Summaries, formerly known as PRODIGY, are part of the National Library for Health and provide a source of evidence-based information and practical know-how relating to the common conditions managed in primary care.

The Clinical Knowledge Summaries website (cks.library.nhs.uk/) is part of the National Library for Health and has been developed from the previous PRODIGY website. Clinical Knowledge Summaries is primarily designed for primary care practitioners (doctors, nurses, pharmacists, medical, nursing and pharmacy students and medical librarians), but it is also useful for other healthcare professionals, and for patients.

The homepage's (Fig. 1) easy-to-read layout contains a clear topic structure, providing topic areas from allergies to women's health, and includes a section on oral health. The latter (Fig. 2) has seven topic areas:

- Aphthous ulcer

- Candida - oral

- Dental abscess

- Gingivitis and periodontitis

- Herpes simplex - oral

- Palliative cancer care - oral

- Wisdom teeth removal (NICE -National Institute for Health and Clinical Excellence)

The navigation structure of the oral health pages is such that it allows easy access to other topic areas. Each clinical topic is divided into a number of sections (Fig. 3).

In the right clinical topic? This outlines the topic scope and applicable age range.

How up-to-date is this topic? This provides information on changes to this topic area, access to previous versions and links to and updates of the evidence. It also provides information on when the next revision is planned.

Background information This section provides relevant definitions and epidemi-
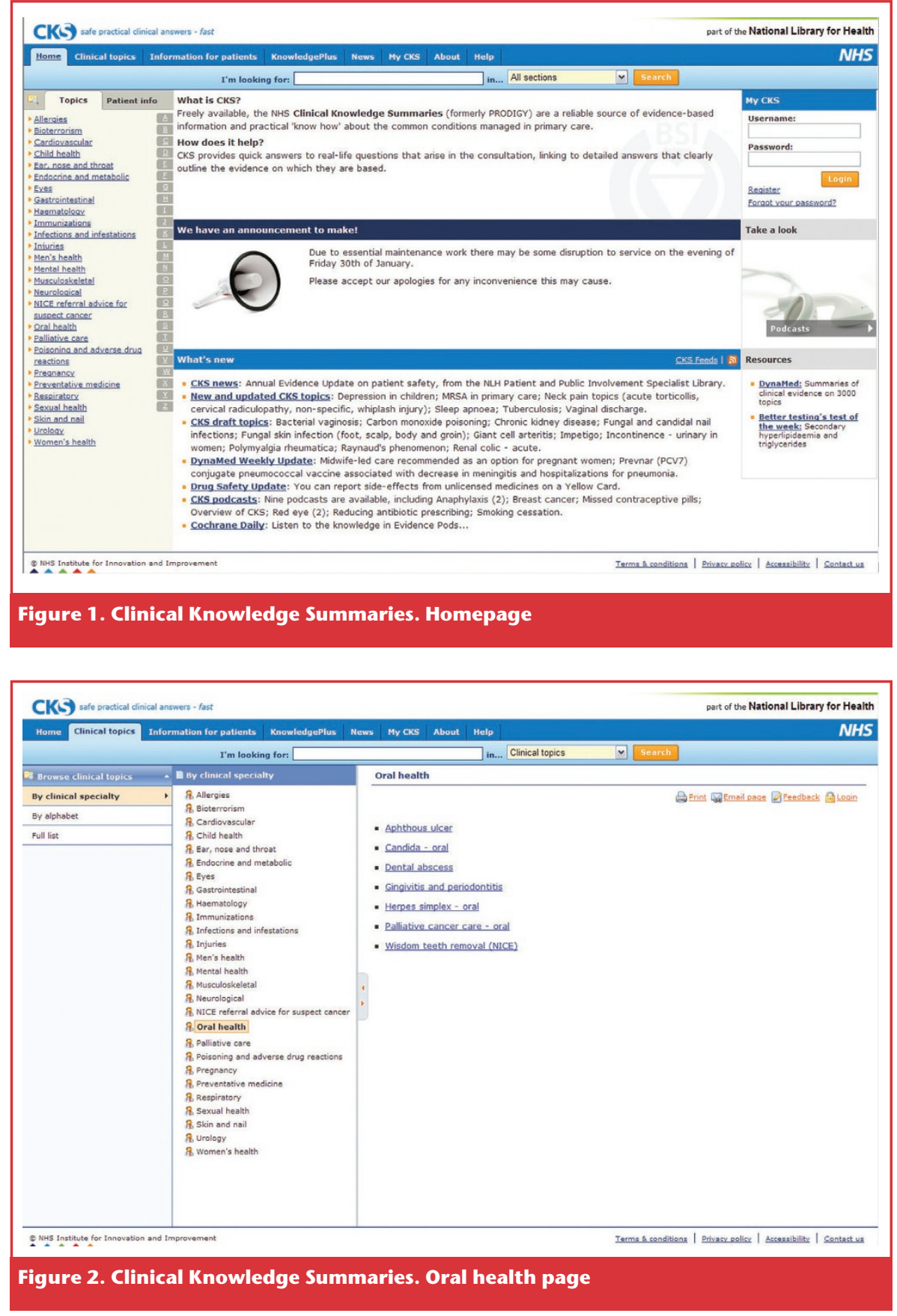
ology, together with causes and risk factors, complications and prognosis.

Making a diagnosis Here, relevant symptoms, signs, investigations and differential diagnoses are provided.

Management The management section highlights treatment goals and outcome measures. It provides "quick answers" for the topic area based around clinical scenarios, which can be used during a consultation. More detailed management information is provided in "detailed answers" for the topic area. The management section also includes prescribing information, although more detailed prescribing information is included within the "Drugs in this topic" section.

Evidence This includes a discussion of the underlying evidence base for the topic area. References are listed along with links to free full-text articles accessible to NHS users in England and Wales

Drugs in this topic Listings of all recommended drugs in this topic together with prescribing information.

Leaflets for patients Contains a range of resources suitable for patients.

Knowledge Plus This section contains relevant links to related information in the National Library of Health.

View the whole topic Contains all the information provided in the various sections

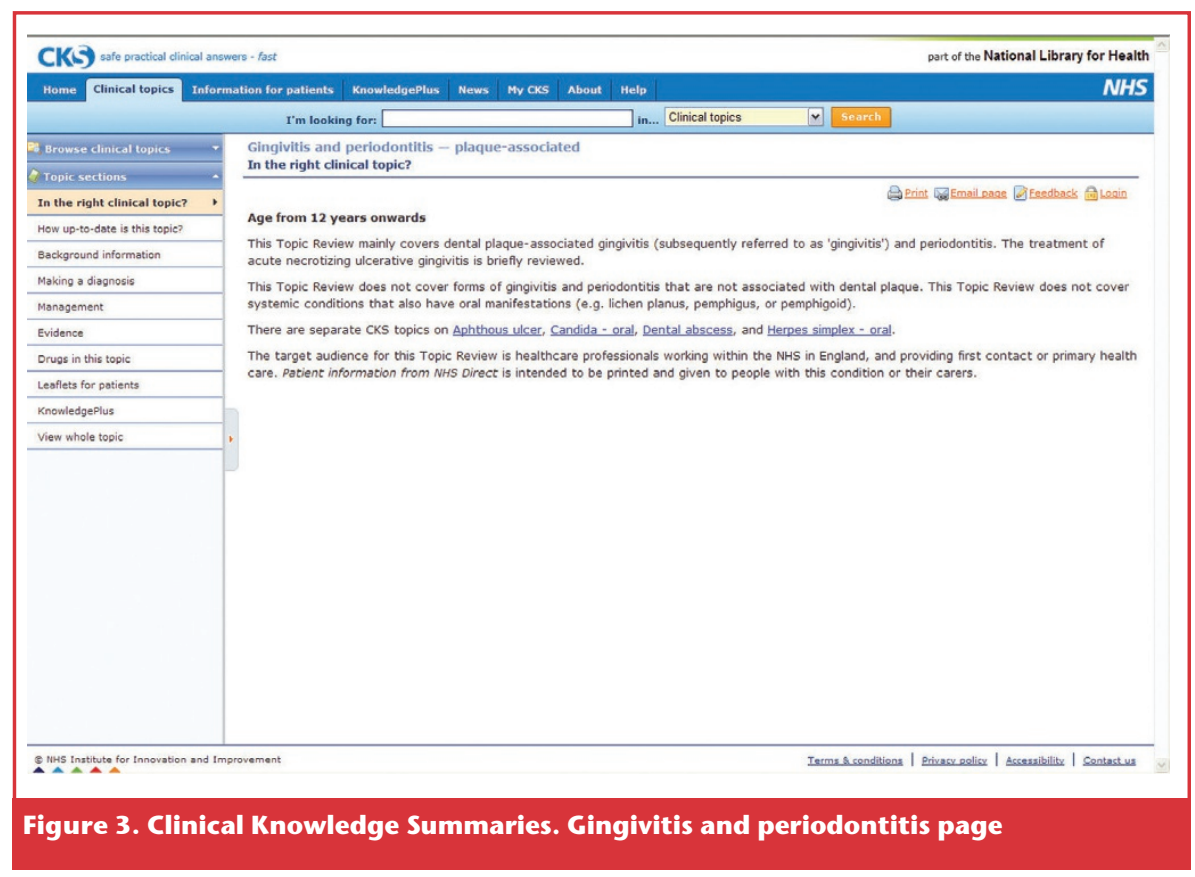

noted above, providing the opportunity for it to be printed out.

NHS, National Health Service.

This section of the National Library of Health (www.library.nhs.uk/) is not primarily intended for a dental audience, but it nonetheless contains useful information for the dental team. The material has been gathered is a systematic manner and regular updates are scheduled, so it can be recommended as an easily accessible overview of the management of these common primary

Evidence-Based Dentistry (2009) 10, 118-119. doi:10.1038/sj.ebd.6400690 care dental problems. 